\title{
First Results on Clusters Observed by XMM/EPIC
}

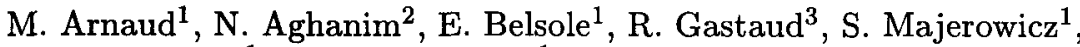 \\ D.M. Neumann ${ }^{1} \&$ J.L. Sauvageot ${ }^{1}$ \\ 1. CEA/DSM/DAPNIA/SAp Saclay, 91191 Gif-sur-Yvette, France \\ 2. IAS-CNRS, Université Paris Sud, 91405 Orsay Cedex, France \\ 3. CEA/DSM/DAPNIA/SEI Saclay, 91191 Gif-sur-Yvette, France
}

\begin{abstract}
First spatially resolved spectroscopic observations of the Virgo and Coma clusters made with XMM during the PV phase are presented. The implication for the dynamical state of these clusters are briefly discussed.
\end{abstract}

\section{Introduction}

The XMM satellite (Jansen et al. 2001), an ESA science mission, has been launched in December 2000. The specificity of XMM lies in the exceptional collecting area of its X-ray telescopes. The EPIC instrument (Turner et al. 2001) on board XMM combines a high sensitivity with good spatial resolution (FWHM $=8 "$, better than $\mathrm{ROSAT} / \mathrm{PSPC})$ and spectral resolution $(\Delta E=60-140$ $\mathrm{eV}$, better than $\mathrm{ASCA})$, on a wide energy range $(0.1$ to $12 \mathrm{keV})$. It is thus specially well suited to study cluster temperature structure, a key information to understand the dynamical state of clusters and merger events (e.g. Schindler \& Müller, 1993). We give here two examples based on PV observations of Virgo (Belsole et al. 2001) and the Coma cluster (Arnaud et al. 2001, Neumann et al. 2001, see also Briel et al. 2001). Full discussions can be found in the papers referenced above, together with references to previous related works.

\section{Virgo}

An image of the substructure in the central part of Virgo, around M87 is presented in Fig. 1. After subtraction of the diffuse emission, the eastern and south-western extensions, related to the radio lobes of M87 and already seen with ROSAT, stand out clearly. A precise spectroscopic analysis could be made with XMM/EPIC. It establishes unambiguously the thermal nature of the X-ray emission, and the spectra permit less than $0.01 \%$ of the output to be power-law in nature, as would be expected from inverse Compton emission. The extensions have a significantly lower temperature $(k T=1.5 \mathrm{keV})$ than the surrounding ambient medium $(k T=2.2 \mathrm{keV})$. The Churazov et al. (2000) framework seems to qualitatively explain the XMM results for the eastern arm but fails to describe the misalignment between $\mathrm{X}$-ray and radio lobes. 


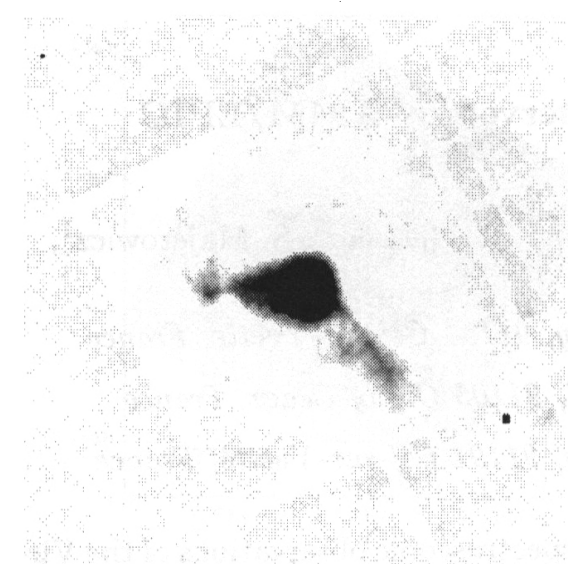

Figure 1. Combination of XMM/EPIC/MOS1 and MOS2 0.2$10 \mathrm{keV}$ of the asymmetric extended $\mathrm{X}$-ray arms; north is up, east is to the left. A model of the M87 extended halo, obtained using wavelet analysis, has been subtracted. The image covers a field of view of 17 arcmin.

\section{Coma}

\subsection{The Central part of Coma}

A temperature map of the central part $\left(r<20^{\prime}\right.$ or $1 / 4$ virial radius) of the Coma cluster is presented in Fig. 2 with the X-ray image substructures overlayed. The temperature distribution around the two central galaxies is remarkably homogeneous $\left(r<10^{\prime}\right)$, suggesting that the core is actually in a relaxed state.

At larger distance from the cluster center we do see evidence for recent matter accretion. The presence of a cool area (Donnelly et al. 1999) in the South-East is confirmed. It generally coincides with the filamentary substructure originating near NGC 4911 and NGC 4921 and is probably due to gas stripped from an infalling group associated with these galaxies.

The XMM temperature map reveals for the first time a hot front in the South-West, just ahead of the excess emission that is seen at the edge of the MOS mosaic and which extends further away towards NGC4839 (see Briel et al. 2001). It is situated roughly at the boundary of the group associated with this galaxy, as defined from the optical and is perpendicular to the direction connecting the center of Coma and NGC4839. This temperature structure is likely to be due to adiabatic compression, caused by the infall of matter associated with the NGC4839 group.

\subsection{The NGC4839 group falling onto the Coma cluster}

Fig. 3 shows the galaxy group around NGC4839, which lies at a projected distance to the Coma cluster center of $1.6 h_{50}^{-1} \mathrm{Mpc}$. The complex temperature structure around NGC 4839 (see the hardness ratio map, the structures there were confirmed by direct spectroscopic analysis) is consistent with simulations of galaxies falling into a cluster environment (Stevens et al. 1999). There are indications of a bow shock and of ram pressure stripping around NGC4839. Furthermore the XMM data reveal a displacement between NGC4839 and the center of the hot gas in the group of about $300 h_{50}^{-1} \mathrm{kpc}$. With a simple approximation this displacement can be explained by the ram-pressure force originating from the infall, which acts much stronger on the group gas than on the galaxies. 


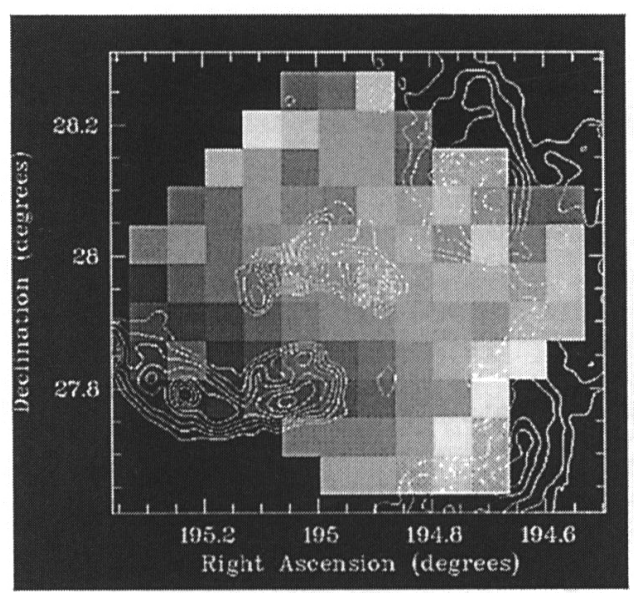

Figure 2. Color coded temperature map from XMM/EPIC/MOS data. Note the hot front in the south-west (white) and the cold region in the South-East (dark). The isocontours overlayed are the residuals (in $\sigma$ ) after subtracting the best fit 2-D $\beta$ model to the $\mathrm{X}$-ray image.

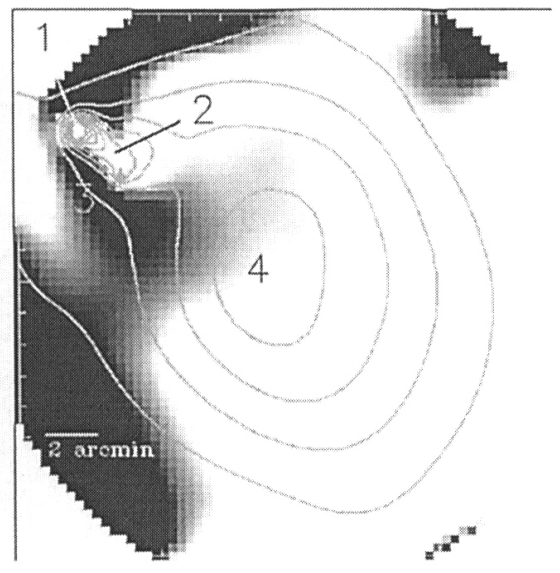

Figure 3. The background subtracted hardness ratio image of NGC4839 group in the bands $2 .-5.0 \mathrm{keV}$ and $0.5-$ $2.0 \mathrm{keV}$. Note the hot region South of NGC4839 (dark) and the cold tail behind the galaxy (white). The contours are the image in the band $0.5-2.0 \mathrm{keV}$ obtained from a wavelet filtering.

Acknowledgments. The authors thank the team of Coma and Virgo Performance Verification observations.

\section{References}

Arnaud, M., et al. 2001, A\&A, 365, L67

Belsole, E., et al. 2001, A\&A, 365, L188

Briel, U., et al. 2001, A\&A, 365, L60

Churazov, E., Brüggen, M., Kaiser, C.R., Böhringer, H., \& Forman, W. 2000, ApJ (submitted, astro-ph/0008215)

Donnelly, R., Markevitch, M., Forman, W., Jones, C., Churazov, E., Gilfanov, M. 1999, ApJ, 513, 690

Neumann, D.M., et al. 2001, A\&A, 365, L74

Jansen, F., et al. 2001, A\&A, 365, L1

Schindler, S., Müller, E., 1993, A\&A, 272, 137

Stevens, I.R., Acreman, D.M., Ponman, T.J., 1999, MNRAS, 310, 663

Turner, M.J.L., et al. 2001, A\&A, 365, L110 